\title{
The influence of surface roughness on electronic transport in thin films
}

\author{
G. Reiss and H. Brückl \\ Institut für Angewandte Phystk, Unt ersttit Regensburg, Unuersitatsstrasse 31, D-8400 Regensburg, Germany
}

Received 9 September 1991, accepted for publı ation 10 September 1991

\begin{abstract}
In thin films, the structure of the surfaces considerably influences the transport of conduction electrons. For mesoscopic roughnesses in the range of a few $\mathrm{nm}$, this is due to the varying film thickness, which gives rise to a spatially fluctuating conductance Moreover, microscopic roughnesses can contribute to the scattering of the electrons and therefore additionally enhance the thin-film resistivity. For a quantitatıve understanding of the transport in these systems, a detailed investıgation of the surface roughness combined with measurements of the electronic properties are necessary. Here, we discuss STM imaging of various metal films and the application of these results to the interpretation of electronic thin-film properties. Provided reasonable resolution of STM in the nm range, a good correspondence of STM results with the electrical behaviour of growing metal films can be established Furthermore, a detalled two-d mensional analysis allows for a calculation of the potential on current-carrying thin films On the other hand, this method supplies reliable values for the electronic transport parameters
\end{abstract}

\section{Introduction}

The conductivity of thin films has been frequently discussed in the literature. Whereas semi-classical models used the Boltzmann equation with appropriate boundary conditions [1], more recent contributions [2-4] gave quantuhmechanical models especially for the thickness depencience of the conductivity. In these discussions, special regard must be naturally taken on the surface scattering of the conduction electrons which is the main reason for the increase of the resistivity of thin films. In ref. [4], it was suggested, that a possible variation of the electron density with thickness could additionally influence the conductivity.

The main purpose of these discussions is the evaluation of reliable transport parameters from the thickness dependent conductivity $\sigma(d)(d$ : film thickness). In order to obtain these values, the theoretical curve must be fitted to the experiment by a variation of usually at least three parameters, one of them representing the surface profile. This, however, usually was performed in one dimenrion without knowledge of the real surface. Therefore, a detailed STM analysis of the thin-film suffaces can considerably improve these investigations. Since STM directly images the surfaces, the discussion of the conductivity can be extended to two dimensions. With this treatment the distribution of the electrostatic potential on current carrying thin films can be additionally estimated. The precondition for these evaluations, however, are reliable results of STM imaging.

\section{Surface imaging}

The imaging of the thin-film surfaces was nerformed with SiM under ambient conditions [5] The materials have been $\mathrm{Pt}, \mathrm{Au}, \mathrm{N}$, $\mathrm{Co}$ and $\mathrm{Cu}$. In order to evaluate possible influences of oxidation, $\mathrm{Ni}$ and $\mathrm{Cu}$ was partly covered with a 1-2 nm thick Au overlayer. In the case of Ni, STM revealed identical surface features without remark- 
able signs of oxidation on the unprotected samples. On pure $\mathrm{Cu}$, reliable imaging was possible only for a few hours after the removal of the films from the UHV chamber. The Cu films protected by $\mathrm{Au}$, however, exhibited the same surface features and allowed stable tunneling for considerably longer time.

As mentioned in the introduction, the resolution of $\mathrm{nm}$ features is of special interest for an application of STM results to electronic transport. This point has been already stressed in refs. $[6,7]$ : based on the STM image anci a properly evaluated tip shape, the real surface can be reconstructed except those parts, the tip was not in tunneling contact with during scanning the surface. The numerical procedure proposed in ref. [8] has been recently reformulated in terms of Legendre transforms [9]. In the recalculated image, the parts which had not been reached by the tip remain as "black hoies". Since the amount of black holes obviously is directly related to the obtained height resolution, we show in fig. 1a a moiel surface consisting of semi-elliptical islands. If the tip indicated in fig. 1a scans this surface, the resulting STM profile corresponds to the line shown in fig. $1 \mathrm{~b}$. Using the definitions of the height resolution indicated in fig. 1, the dependence of this quantity on the amount of surface without tunneling contact to the tip can be estimated. The result of this calculation is shown in fig. 2 for different ratios $a=R / H$.

As can be seen from this estimate (fig. 2), the obtained height resolution depends very critically on the amount of unresolved surface area. Only a few percent of black-hcle surface can cause a considerable underestimate of the roughness. For the further discussion, we therefore used only

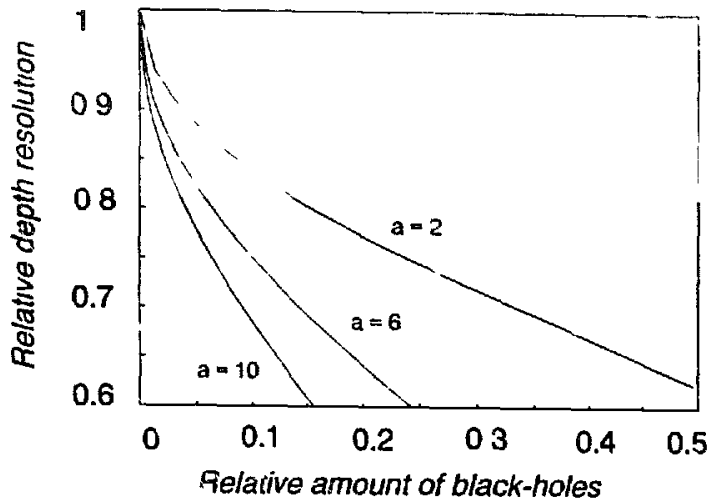

Fig. 2 The height :esolution of STM images as a function of the surface area without tunneling contact to the tip

images with a black-hole area equal or less than $1 \%$ of the whole surface.

If, however, the precondition concerning the resolution is fulfilled, STM clearly can provide reliable surface profiles for the discussion of the electronic transport properties.

\section{The thickness dependence of the resistivity}

For the evaluation of the transport parancters, we use the model of Tešanović et al [2]. Here, the thickness dependence of the conductıv1ty is given by:

$$
\begin{aligned}
& \sigma_{\text {lin }}\left(d(x), l_{x} h^{2}, \sigma_{x}\right) \\
& =\frac{\sigma_{x}}{n_{\mathrm{c}}} \sum_{n=1}^{n_{\mathrm{c}}}\left[1+\frac{l_{x} h^{2} k_{\mathrm{f}}^{2}}{6 \pi d(x)}\left(\frac{n}{n_{\mathrm{c}}}\right)^{2}\right]^{-1} .
\end{aligned}
$$

where the sum includes the occupied subbands (index $n), d(x)$ is the local film thickness, $k_{\mathrm{f}}$ is the Fermi wavevector, $\sigma_{x}$ is the conductivity of
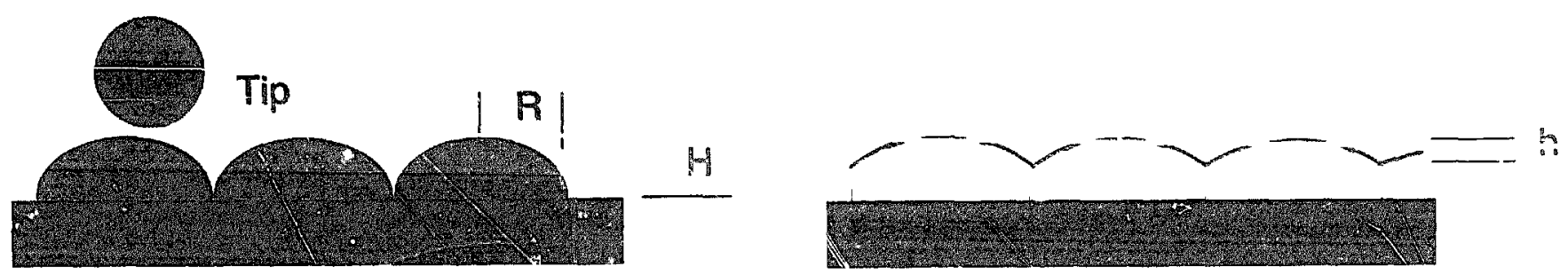

Fig 1 (a) Model surface for estımating the height resolution of STM (b) STM profile corresponding to the tip/hurface combination of (a). The height resolution is defined as the ratio $h / H$ 


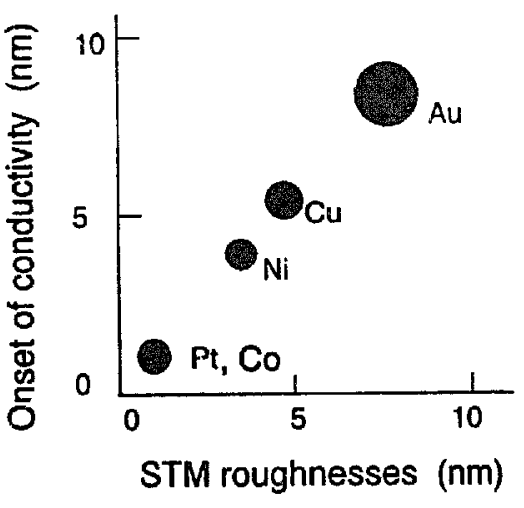

Fig 3. Onset thickness of the ohmic conductivity of films growing on glass substrates at room temperature compared with the correspondin; roughness found by STM. The rauius of the dots corresponds to the spread of the experimental datd

infinitelv thick materral and $l_{x} h^{2}$ is the product of the intrinsic electronic mean free path and the microscopic oughness of the surface. Roughnesses considerably larger than the Fermi wavelength can be included by averaging over a varying film thickness $d(x)$ similar to the discussions given in refs. $[4,7,10]$.

The application of STM results can be performed in one dirmension simply by using a corresponding one-dimensional roughuess distribution The preconditions and results of this ireatment have been already discussed in ref [7] for thin $\mathrm{N}_{1}$ films. In order to demonstrate the relevance of the method for different materials, fig. 3 shows the correlation of roughnesses estimated by two methods:

First, the roughness can be simply obtained via highly resolved STM images. Second, the mean roughness should correspond to the thickness of the growing film, where the onset of the ohmic conductivity can be found [7] (see eq. (1)).

From fig. 3, a suprisingly good agreement between these values of the thin film roughress can be established for different metals in a range from about 1 to $10 \mathrm{~nm}$.

On the other hand, the one-dimensional model cannot reproduce the conductivity especially for very smail thicknesses [ij. Tinis is due iu the lack of percolation, 1.e, withın this treatment the conductivity approaches zero at a thickness corresponding to the maximum corrugation found by
STM. In orciel to achieve a better description, we thus . xtenced our discussion to two dime asions.

For this purpose, the film was modeled as a $(128 \times 128)$ networks of resistors, each of which represents a small portion of the integral film resistance. The corresponding local conductances can be obtained from eq. (1) in combination with the local thicknesses supplied by the STM image. In order to obtain the conductivity, the voltage has to be fixed at two edges of the STM image. The resulting voltages $V_{l}$ at node $i$ of the network can be obtained selfconsistently using the local conductances $g_{i,}$ between node $i$ and node $j[11]$ : $V_{l}=\sum_{j=1}^{4} \frac{V_{j}}{g_{t \jmath}}, \sum_{j=1}^{4} g_{\imath \jmath}$

This self-consistent formalism quickly converges towards the distribution $\left\{V_{l}\right\}$ of the potential on the thin-film surface. Using this potential, the current can be evaluated using the local conductances defined before. From these values of current and voltage, the integral conductivity can be obrained in the usual way.

In fig. 4, we show the results of this two-dimensional estimate together with the simpler one-dimensional model and the experimental results for the resistivity of a $N$ thin film

As can be seen from fig. 4, the one-dinensional fit approaches the experimental results at a thickness of about $7 \mathrm{~nm}$. The maximum depth of

\section{0}

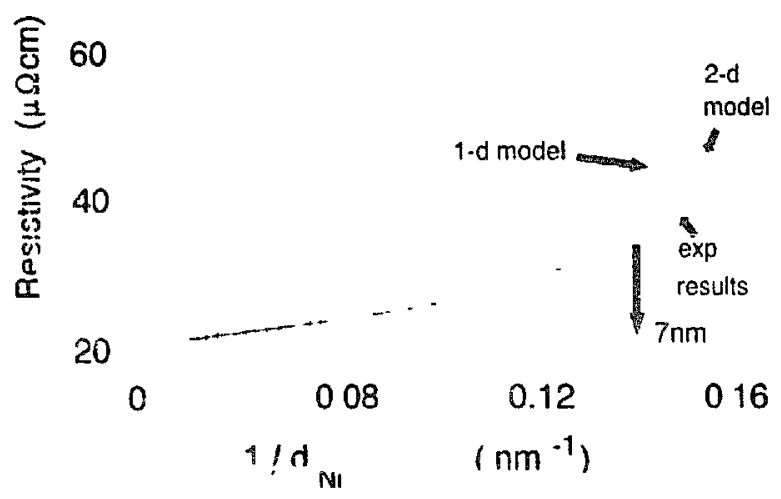

Fig a Results of litting the theoretical expressions for the thicknes-dependent thin-film conductivity to the experimental cune 
the bumps found by STM on this surface was about $6 \mathrm{~nm}$ [7]. On the other hand, the two-dimensional treatment can reproduce the experimental curve down to much smaller thicknesses As already mentioned, this is due to the percolative behaviour for thicknesses smaller than the maximum roughnesses, which can be treated solely by a two-dimensional description.

The bulk resistivities $\rho_{x}$ obtained from the fitting calculations are 19.5 and $21 \mu \Omega \mathrm{cm}$ for the one- and two-dimensional case, respectively. For the product of the mean free path with the microscopic surface roughness, we obtained $1.25 \mathrm{~nm}^{3}$ (1D) and $1.4 \mathrm{~nm}^{3}$ (2D). Since the two-dimensional treatment is more realistic, the mean free path therefore seems to be sumewhat larger than the values obtained from the one-dimensional discussion. Nevertheless, already the simple onedimensional model reveals rather correct values for this fundamental transport parameter.

The two-dimensional model, however, can supply more information: As discussed before, the distribution of the electrostatic potential on the current-carrying thin film must be calculated for fitiing the theory to the experiment. On the other hand, this distribution itself is of considerable interest In fig. 5 we show the original STM topography of a $10 \mathrm{~nm}$ thick Ni film (tıg 5a) and the corresponding calculated distribution of the local electrostatic field defined as the magnitude of the gradient of the local potential. A good correspondence of the topographical features with the local field can be established: at locations of small film thickness, the local field can be enhanced by a factor two compared with the mean field.

Fig. 5 therefore shows, that a rather inhomogeneous distribution of the potential on rough thin films can be expected due to the spatially varying conductances. On the other hand, the drop of the potential itself is not as inhomogencous as found by potentiometric STM measurements on polycrystaliine thin films [12,13], Thus, it seems to be necessary to include grain boundary scattering in the discussion of the experimentally evaluated potential [14].

\section{Conclusions}

In this contribution. we presented a two-dimensional treatment of the thickness-dependent
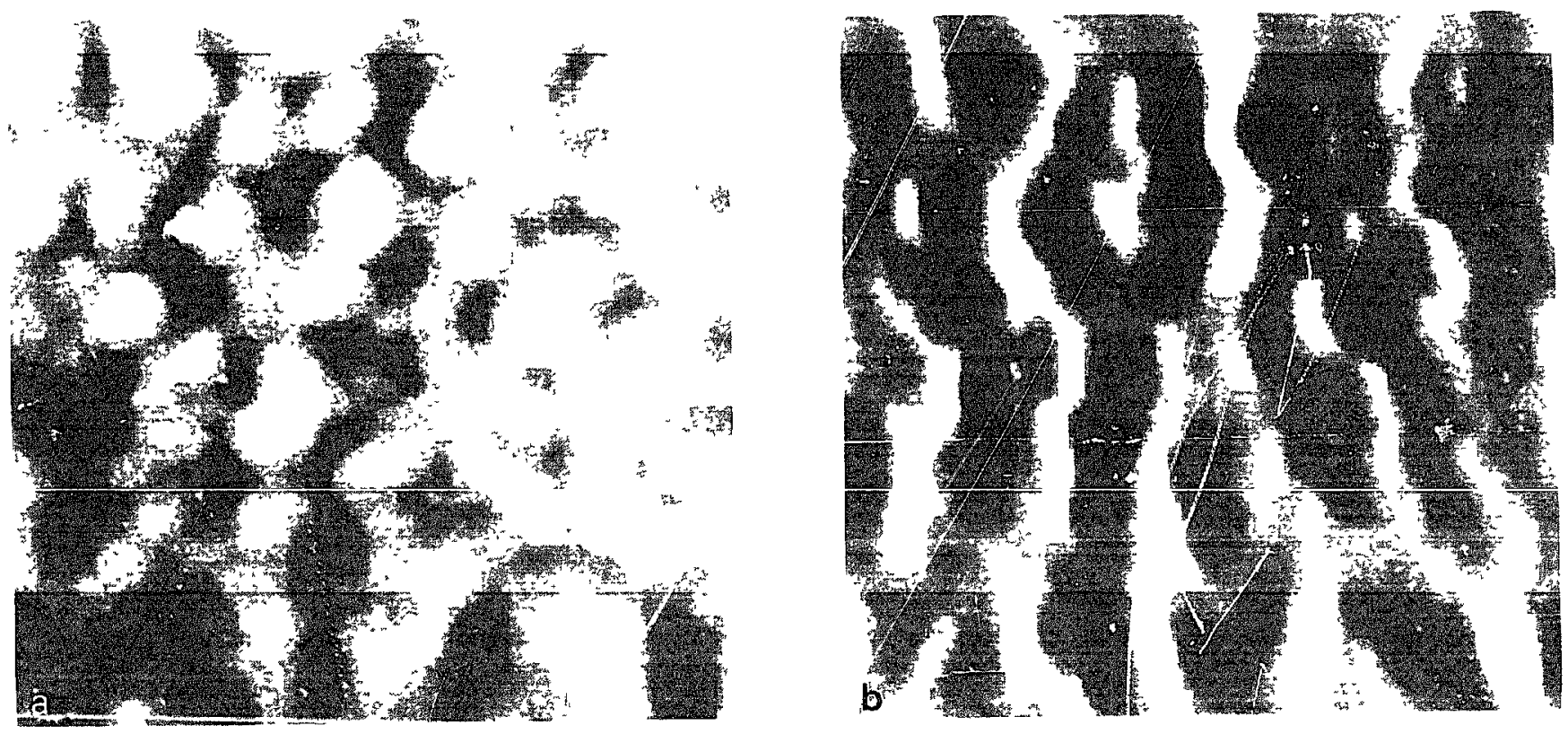

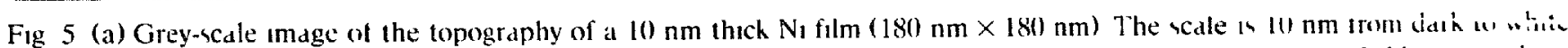
(b) The magnitude of the local feald calculated for the surface togography shown in (a) Wnite correspond to a field twice an large d. ditk 
thın-film $\mathrm{r}$.sistıvitv. The surface profiles necessary for this purpose can be supplied by highly resolved STM imiges. The STM roughnesses of the surfaces have been shown to correspond very well with the thickness of the onset of ohmic conductivity, i.e., just with the stage of coalescence of the growing film.

A two-dimensional fit of the experimental data of the thickness-dependent thin-film resistivity can be performed using a selfconsistent resistor network model and the topography supplied by STM. Although th.e realistic two-dimensional model supplies slightly different values, the results for the transport parameters agree rather well with former one-dimensional treatments $[7,10]$. In contrast with this, however, the extended discussion can include the stage of percolation, i.e.. using this formalism, network structures can be treated. Moreover, this formalism naturally supplies the distribution of the potential on a current-carrying thin film. This turns out to be rather inhomogeneous as soon as the roughness becomes comparable to the film thickness. The potential obtained, however, is still smoother thas observed by potentiumetric STM measure- ment. Therefore it seems to be necessary to include additionally grain boundary scattering in order to explain these results.

\section{References}

[1] K Fuchs, Proc Cambridge Phil. Soc. 34 (1938) 100.

[2] Z Tešanović, MV Jarić and S. Maekawa, Phys. Rev Lett 57 (1986) 2760

[3] D. Caleckı and G Fishman, Surf Scı 229 (1990) 110.

[4] N Trivedı and N W Ashcroft, Phys. Rev B 38 (1988) 12298.

[5] J Vancea, G. Reıss, F. Schneider, K Bauer and H Hoffmann, Surf. Scı 218 (1989) 108.

[6] G. Reiss, H Brückl, J Vancea, R. Lecheler and E. Hastreiter, J, Appl Phys. 70 (1991) 523

[7] G. Reiss, E. Hastreiter, H. Bruckl and J Vancea, Phys. Rev, B 43 (1991) 5176

[8] G. Reıss, F. Schneider, J. Varcea and H. Hoffmann, Appl. Phys Lett 57 (1990) 867

[9] D. Keller, Surf Sc1. 253 (1991) 353

[10] U. Jacob, J Vancea and H. Hoffmann, Phys, Rev B 41 (1990) 11852

[11] S. Kirkpatrick, Rev. Mod Phys 45 (1973) 574

[12] J P Pelz and R H Koch, Rev. Sc Instrum. 60 (1989) 301

[13] J P Pelz and R H. Koch, Phys Rev B 41 (1990) 1212

[14] C S Chu and R S Sorhello, Phy's Rev B 42 (1990) 4928. 